etal muscle vasodilation is nitric oxide dependent. A novel action of insulin to increase nitric oxide release. J. Clin. Invest. 94:1172-1179.

12. Trovati, M., et al. 1994. Insulin increases guanosine$3^{\prime}, 5^{\prime}$-cyclic monophosphate in human platelets. A mechanism involved in the insulin anti-aggregating effect. Diabetes. 43:1015-1019.

13. Aljada, A., and Dandona, P. 2000. Effect of insulin on human aortic endothelial nitric oxide synthase. Metabolism. 49:147-150.

14. Zeng, G., and Quon, M.J. 1996. Insulin-stimulated production of nitric oxide is inhibited by wortmannin. Direct measurement in vascular endothelial cells. J. Clin. Invest. 98:894-898.

15. Esposito, K., et al. 2002. Inflammatory cytokine concentrations are acutely increased by hyperglyce- mia in humans: role of oxidative stress. Circulation. 106:2067-2072.

16. Aljada, A., et al. 2004. Glucose intake induces an increase in activator protein 1 and early growth response 1 binding activities, in the expression of tissue factor and matrix metalloproteinase in mononuclear cells, and in plasma tissue factor and matrix metalloproteinase concentrations. Am. J. Clin. Nutr. 80:51-57.

17. Furnary, A.P., et al. 2003. Continuous insulin infusion reduces mortality in patients with diabetes undergoing coronary artery bypass grafting. J. Thorac. Cardiovasc. Surg. 125:1007-1021.

18. Jeschke, M.G., Klein, D., and Herndon, D.N. 2004. Insulin treatment improves the systemic inflammatory reaction to severe trauma. Ann. Surg.
239:553-560

19. Krinsley, J.S. 2004. Effect of an intensive glucose management protocol on the mortality of critically ill adult patients. Mayo Clin. Proc. 79:992-1000.

20. Jeschke, M.G., Klein, D., Bolder, U., and Einspanier, R. 2004. Insulin attenuates the systemic inflammatory response in endotoxemic rats. Endocrinology. 145:4084-4093.

21. Stentz, F.B., Umpierrez, G.E., Cuervo, R., and Kitabchi, A.E. 2004. Proinflammatory cytokines, markers of cardiovascular risks, oxidative stress, and lipid peroxidation in patients with hyperglycemic crises. Diabetes. 53:2079-2086

22. Langouche, L., et al. 2005. Intensive insulin therapy protects the endothelium of critically ill patients. J. Clin. Invest. 115:2277-2286. doi:10.1172/JCI25385.

\title{
You are right too!
}

\author{
Joseph L. Witztum
}

Department of Medicine, University of California San Diego, La Jolla, California, USA.

\begin{abstract}
Macrophage scavenger receptors, such as CD36 and class A scavenger receptor (SR-A), have previously been thought to play a central role in foam cell formation and atherogenesis by mediating the uptake of oxidized LDL. In this issue of the JCI, Moore et al. report that $\mathrm{Apoe}^{-/-}$mice deficient in either CD36 or SR-A did not have less atherosclerosis at the level of the aortic valve than did wild-type $A p o e^{-/-}$mice (see the related article beginning on page 2192). In contrast, similar studies by previous investigators found that deletion of these receptors decreased atherogenesis. The reasons for the different results are not known, but these data suggest that the role of these receptors in atherogenesis remains unresolved.
\end{abstract}

In the small villages of eastern Europe, the rabbi was the undisputed leader of the Jewish people of his village. Not only was he the master of religious wisdom and law, but he often served as the arbiter of civil disputes as well. There is told the tale of 2 individuals who had a major dispute and agreed to go to the rabbi for resolution. The first party to the dispute came to the rabbi and carefully outlined his side of the argument. The rabbi listened intently and finally said, "My friend, you are right." The man went away satisfied. Later in the day, the other party to the dispute arrived and told the rabbi his side of the issue. The rabbi again listened carefully, was impressed with the arguments, and replied after some thought, "You are right." Later, the rabbi's wife, who had overheard the rabbi's conversations with both men, said to him, "Rabbi, you

Nonstandard abbreviations used: LDLR, LDL receptor; mmLDL, minimally modified LDL; OxLDL, oxidized LDL; SR-A, class A scavenger receptor; SR-BI, class B, type I scavenger receptor.

Conflict of interest: The author has declared that no conflict of interest exists.

Citation for this article: J. Clin. Invest. 115:2072-2075 (2005). doi:10.1172/JCI26130. told both the first party and the second party that they were right. How can this be?" To which the rabbi replied, "And you are right too!"

So we too are faced with a dilemma. In the current issue of the JCI, Moore et al. report that $A p^{-/-}$mice deficient in either class A scavenger receptor (SR-A) or CD36 and fed an atherogenic diet showed a reduction in peritoneal macrophage lipid accumulation in vivo; however, there was no decrease in atherogenesis (1). To put these results into perspective, previous reports by equally reputable investigators have provided convincing data supporting a role for these 2 scavenger receptors in the generation of foam cells and the development of atherosclerosis (2-4). These data are particularly strong for the CD36 receptor. Furthermore, a recent study in which bone marrow from either $\mathrm{Cd} 36^{-/-}$or wildtype mice was transplanted into cholesterol-fed Apoe-/- mice demonstrated that the absence of CD36 provided substantial protection against lesion formation and the reintroduction of $\mathrm{CD} 36^{+}$macrophages increased lesion area (5). The data strongly suggest that CD36 expressed on macrophages is an important contributor to foam cell formation and atherosclerosis. So like the rabbi's wife, we are left to ask, "How can this be?"

\section{Analysis of the study}

Let us first examine the current study by Moore et al. (1). There are many aspects of their data that support their conclusions. The authors have generated a carefully defined genetic model that was essentially congenic to the $\mathrm{C} 57 \mathrm{BL} / 6$ background. The authors began with the same $\mathrm{Sra}^{-/-}$mice generated by Kodama and colleagues (2) and the $C d 36^{-/-}$mice generated by Febbraio and Silverstein (4) and backcrossed them to be $99 \%$ congenic with the C57BL/ 6 background. In this respect, the mice studied by Moore et al. did differ from some, but not all, of the animals used in similar experiments. It is of course well known that different murine strains have differing susceptibilities to atherosclerosis and, as even a single gene product can influence this susceptibility, it is conceivable that some of the prior experiments were influenced by the presence of unrecognized diseasemodifying gene(s) that were not a factor in the experiments presented here (1). However, the results of the bone marrow transplant study cited above (5) suggest that if such disease-modifying genes are involved, they would need to be primary products of bone marrow-derived cells.

Despite the robustness of the observation that atherosclerosis was not decreased at the aortic valves in both $\mathrm{Sra}^{-/-} \mathrm{Apoe}^{-/-}$and $\mathrm{Cd} 36^{-/-} \mathrm{Apoe}^{-/-}$mice, a number of issues need to be considered in interpreting these 


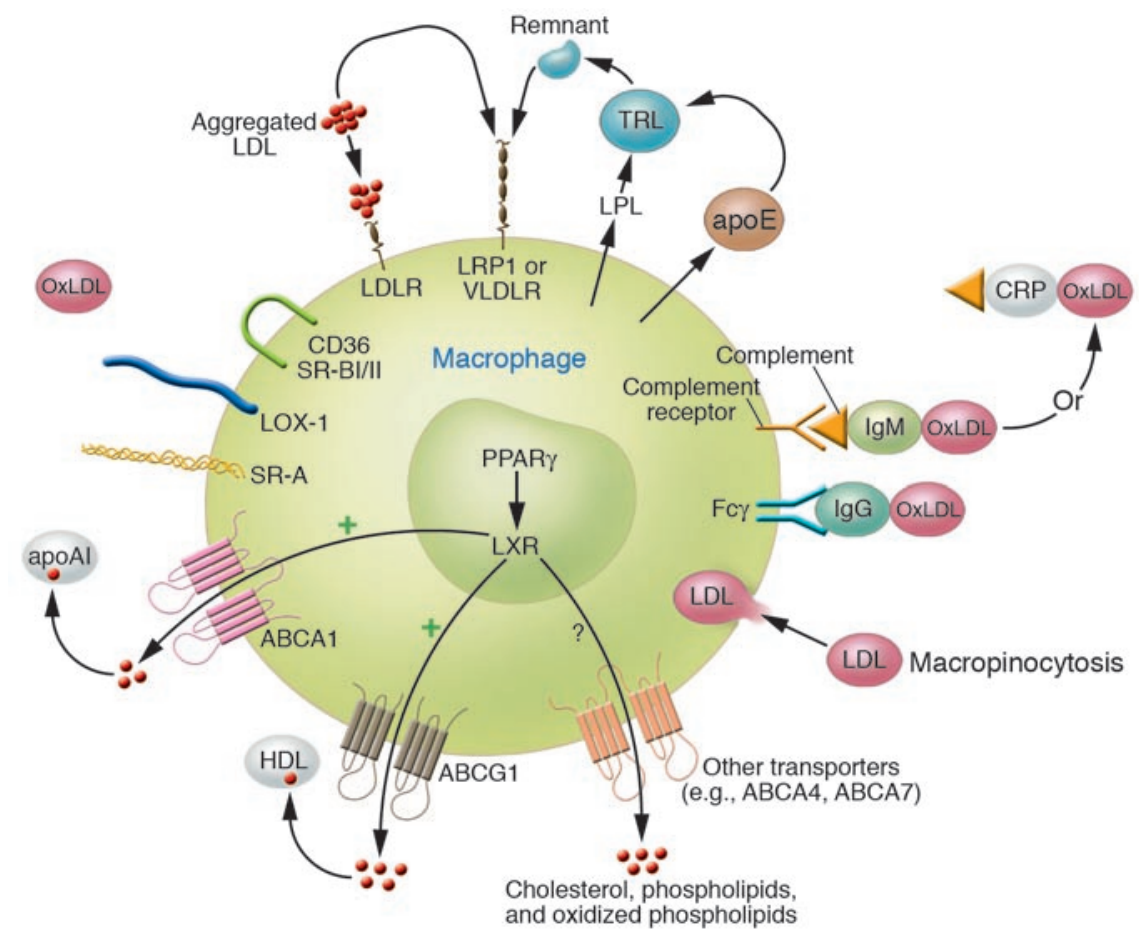

Figure 1

Theoretical mechanisms by which macrophage foam cells could be generated. Excess cholesteryl ester accumulation could occur because of the following: (a) enhanced uptake of native or modified lipoproteins or lipids; (b) alterations in intracellular cholesterol metabolism; (c) failure of export systems to maintain cholesterol homeostasis; or (d) combinations of the aforementioned mechanisms. Scavenger receptors expressed on the surface of macrophages that may contribute to the uptake of modified lipoproteins include SR-A, CD36, SR-BI, PSOX, MARCO, and lectin-like OxLDL receptor 1 (LOX-1). LDLR and LDLR-related protein 1 (LRP1) can mediate uptake of aggregated LDLs (including native or enzymatically modified LDLs), and secreted apoE can bind to such complexes and facilitate uptake, particularly via LRP1. LDL/lgG complexes can be internalized via Fc receptors, and lipoproteins bound by IgM or C-reactive protein (CRP) can in turn bind complement and undergo enhanced binding via complement receptors. Secreted lipoprotein lipase (LPL) can hydrolyze triglyceride-rich lipoproteins (TRLs), generating FFAs that can enter the cell and stimulate cholesteryl esterification. Residual remnant lipoproteins can be taken up via LRP1 or even LDLR or VLDLR. ABC transport proteins $A B C A 1$ and $A B C G 1$ and probably others, such as $A B C A 4, A B C A 7$, and SR-BI/II, can mediate the export of cholesterol, phospholipids, and/or oxidized phospholipids to apoAI, HDL, or other acceptors. Transcription of these transporters is regulated by PPAR $\gamma$ and liver $X$ receptor $(L X R)$, and they are also subject to posttranscriptional regulation by apoAI. Finally, uptake of native, modified or aggregated LDL could occur via stimulated macropinocytosis, independent of or in association with receptor-mediated uptake. Undoubtedly, many other factors influence net cholesterol accumulation.

data (1). First, the plasma cholesterol levels were nearly $40 \%$ higher in the $\mathrm{Sra}^{-/-} \mathrm{Apoe}^{-/-}$ and $\mathrm{Cd} 36^{-/-} \mathrm{Apoe}^{-/-}$male mice (significant) and $20 \%$ higher in the female double-KO mice (not significant) in comparison with those of sex-matched Apoe $e^{-/-}$animals. Many research groups have shown a positive relationship between lesion formation and plasma cholesterol levels in such mice $(6,7)$. Conceivably, any decrease in lesion formation as a result of scavenger receptor deletion might have been overcome by the substantially higher cholesterol levels in lesion formation at specific sites have been noted (8). A third point of potential importance is that the extent of atherosclerosis was examined at only 1 time point - after 8 weeks on the Western-type diet. By comparison, other published reports of the impact of scavenger receptor deficiency on atherosclerosis in mice have examined later time points. In this regard, a recent report showed that the presence of class B, type I scavenger receptor (SR-BI) in macrophages was proatherogenic at the earliest phase of lesion formation (after 4 weeks on a Western-type diet) but played an overall protective role in later stages of lesion formation (after 9 and 12 weeks) in $\mathrm{Ldlr}^{/-}$mice (9). Likewise, in the CD36 bone marrow transplant model described above (5), the extent of atherosclerosis was determined after 12 weeks of a Western-type diet. The most dramatic reduction in lesion formation in the $C d 36^{-/-}$Apoe $e^{-/-}$mice was observed in the aorta, while the decrease at the aortic valve was much less impressive. These examples suggest that, for proper comparisons to be made between studies, we need to look at the impact of interventions at both early and late time points, at different anatomical sites, and in both sexes.

\section{Discrepancy between lipid accumulation in macrophages of the aorta and peritoneum}

Based on their data, Moore et al. (1) suggest that in vivo scavenger receptor-mediated uptake of oxidized LDL (OxLDL) may not be a rate-limiting step in atherosclerosis. They describe their interesting observations that peritoneal macrophages elicited from the cholesterol-fed male $\mathrm{Sra}^{-/-} \mathrm{Apoe}^{-/-}$and Cd36-1-Apoe e $^{-1-}$ mice contained $40-60 \%$ less cellular cholesterol and 70-80\% less cholesteryl ester as compared with those of control Apoe $e^{-/-}$mice. Interestingly, no significant differences in macrophage cholesterol and cholesteryl ester content were observed between female $\mathrm{Sra}{ }^{-/-} \mathrm{Apoe}^{-/-}$and $\mathrm{Cd} 36^{-/-} \mathrm{Apoe}^{-/-}$mice and sex-matched Apoe ${ }^{-/-}$controls. If these scavenger receptors are not at the center of lipoprotein uptake, as Moore et al. suggest, it is unclear why their loss should result in decreased lipid accumulation in peritoneal macrophages (at least in male mice). This suggests that uptake of OxLDL was decreased somewhere in the body to yield these results. Our lab, Moore et al., and others have suggested that peritoneal macrophages from such mice are a model of macrophage behavior in the artery wall (10). However, Moore et al. report that the macro- 
phages in the lesions appeared to be replete with lipid (1). Perhaps the assumption that the pattern of gene expression by peritoneal macrophages reflects that of macrophages within arterial lesions is not valid. Furthermore, in the presence of marked hypercholesterolemia, the relative concentration of extracellular, modified LDL was most likely also equally increased and may have led to saturation kinetics of the existing scavenger receptors. Under such conditions, alternative scavenger receptors could conceivably mediate sufficient uptake of modified LDL, leading to foam cell formation.

There are, in fact, a number of scavenger receptors, in addition to CD36 and SR-A, which could take up OxLDL $(10,11)$. Furthermore, compensatory changes in expressed receptors might overcome the loss of the deleted receptor. For example, minimally oxidized LDL, e.g., minimally modified LDL (mmLDL), itself not a ligand for scavenger receptors, can nevertheless stimulate both mRNA and protein expression of CD36, SR-A, and other receptors on macrophages, leading to enhanced OxLDL uptake that could more than compensate for the absence of the deleted receptor $(12,13)$. The expression of these receptors is influenced by both transcriptional and posttranscriptional regulation $(14,15)$ Indeed, for this reason and many more, the local regulation of scavenger receptor expression in the artery may be different from that in macrophages elsewhere, such as those that appear in the peritoneum. Because in vitro experiments with macrophages from $\mathrm{Cd} 36^{-/-} \mathrm{Sra}^{-/-}$double-KO mice indicated a nearly $80 \%$ decrease in the uptake of OxLDL (16), in vivo experiments in which these mice are crossed into the $A p o E^{-/-}$mouse and then assessed for atherosclerosis are eagerly awaited.

\section{Alternative pathways to produce foam cells}

In analyzing problems we all have the tendency to think of either. . or when most often the answer is both. Thus, the situation with respect to foam cell development and atherogenesis may not simply be that OxLDL uptake is not relevant and that other pathways are more important but that both occur simultaneously. However, for the sake of argument, let us consider alternative mechanisms that do not require scavenger receptor-mediated OxLDL uptake to generate macrophage foam cells. In fact, there are numerous such pathways that can lead to cholesterol accumulation as a consequence of enhanced uptake of lipoproteins or lipids (Figure 1). For example, Khoo et al. first showed that aggregation of native LDL greatly enhanced its uptake via the LDL receptor (LDLR) pathway (17). Indeed, one of the earliest consequences of oxidation of LDL is aggregation, and undoubtedly, some of the enhanced uptake of OxLDL is due to this pathway. Other modifications of LDL that take place in the artery may also induce such aggregated complexes, including enzymatic modifications, for example, via sphingomyelinase and various phospholipases (18). Immunoglobulin-coated LDL may also have enhanced uptake (17), and uptake of such immune complexes has been reported to increase LDLR activity (19). In turn, this could lead to enhanced uptake of native and/or aggregated LDL. Indeed, oxidized phospholipids found in mmLDL and OxLDL have been reported to increase LDLR activity in endothelial cells (20), and conceivably, mmLDL could produce a similar effect in macrophages. As emphasized by Kruth and colleagues, macrophage foam cells can also be generated in vivo following activation by phorbol 12-myristate 13-acetate, which leads to stimulation of native LDL uptake by increased fluid-phase endocytosis via a macropinocytosis pathway (21). Lipoprotein lipase secreted by macrophages can hydrolyze triglycerides in VLDL, leading to enhanced FFA and triglyceride-rich lipoprotein remnant uptake and increased cholesteryl ester accumulation (22). Interference with the regulation of intracellular cholesterol transport or of proteins that export cholesterol from the cells, such as ABCA1 and/or ABCG1, can also lead to increased macrophage cholesterol content $(15,23)$. Under a given set of parameters, for example, in the presence of mild hypercholesterolemia, only a few of these (or other) pathways may be operative and sufficiently contributory to be rate limiting. On the other hand, when plasma cholesterol levels are exceedingly high, for example, it may well be that all of these pathways are operative and each individually sufficient to drive foam cell formation. In this latter scenario, deletion of any one pathway (or even 2) may not influence lesion formation.

\section{Deletion of a gene product may have unexpected consequences}

There is yet another important conceptual idea that needs to be addressed. We all tend to use linear reasoning in evaluating the impact of a gene deletion in the context of atherosclerosis. Thus, we assume that the contribution of a given gene product is linearly related to disease outcome such that deletion of a given scavenger receptor, for example, will result in a predictable decrease in OxLDL uptake and a linear decrease in lesion formation. However, it is not likely that nature operates in such a simple manner, and in fact, a nonlinear response is more likely. Deletion of CD36, for example, may have an impact on many other biological properties in unforeseen ways. CD36 is a classic innate pattern recognition receptor and binds many divergent and seemingly unrelated ligands. It is involved in many functions quite apart from binding and uptake of OxLDL, such as the binding and uptake of apoptotic cells. It may associate with other receptors in the cell, such as Toll-like receptor 2 or certain integrins, to initiate signaling cascades $(24,25)$, and via its ability to internalize a variety of ligands such as FFAs, it may affect many cellular functions. The genetic deletion of CD36 may have led to compensatory responses not yet appreciated that in turn may have affected lesion development in unexpected ways. As SR-A is also such an innate pattern recognition receptor, these possibilities may also hold true.

\section{Conclusions}

Despite all of these comments, it is not clear why the study from Moore et al. (1) yields conclusions different from those of other investigators. So at this time we find ourselves, like the rabbi in our opening story, saying to both the authors of this study and those of preceding studies that came to the opposite conclusion, "You are right." However, like the rabbi's wise wife, we need to continue to ask "How can this be?"

While this controversial study provides an alternative answer to a question believed to have been answered previously, it does not provide an explanation of why the 2 answers differ. Sometimes papers are noteworthy because they provide definitive insight into a fundamentally important issue. On the other hand, sometimes we think things are settled when in reality they are not. When studies such as this are well done, it is important to publish them, as they have an inherent heuristic value. The data presented by Moore et al. (1) will cause all of us to stop and reevaluate and to undertake much more experimentation and thought.

\section{Acknowledgments}

The author thanks Agnès Boullier and Dan Steinberg for many helpful suggestions and comments. 
Address correspondence to: Joseph L. Witztum, Department of Medicine, University of California San Diego, 9500 Gilman Drive, La Jolla, California 92093, USA. Phone: (858) 534-4347; Fax: (858) 534-2005; E-mail: jwitztum@ucsd.edu.

1. Moore, K.J., et al. 2005. Loss of receptor-medi ated lipid uptake via scavenger receptor A or CD36 pathways does not ameliorate atherosclerosis in hyperlipidemic mice. J. Clin. Invest. 115:2192-2201. doi:10.1172/JCI24061.

2. Suzuki, H., et al. 1997. A role for macrophage scavenger receptors in atherosclerosis and susceptibility to infection. Nature. 386:292-296.

3. Babaev, V.R., et al. 2000. Reduced atherosclerotic lesions in mice deficient for total or macrophagespecific expression of scavenger receptor-A. Arterioscler. Thromb. Vasc. Biol. 20:2593-2599.

4. Febbraio, M., et al. 2000. Targeted disruption of the class B scavenger receptor CD36 protects against atherosclerotic lesion development in mice. J. Clin. Invest. 105:1049-1056.

5. Febbraio, M., Guy, E., and Silverstein, R.L. 2004 Stem cell transplantation reveals that absence of macrophage CD36 is protective against atherosclerosis. Arterioscler. Thromb. Vasc. Biol. 24:2333-2338.

6. Freigang, S., Hörkkö, S., Miller, E., Witztum, J.L., and Palinski, W. 1998. Immunization of LDL receptor-deficient mice with homologous malondialdehyde-modified and native LDL reduces progression of atherosclerosis by mechanisms other than induction of high titers of antibodies to oxidative neoepitopes. Arterioscler. Thromb. Vasc. Biol. 18:1972-1982.

7. Skalen, K., et al. 2002. Subendothelial retention of atherogenic lipoproteins in early atherosclerosis.
Nature. 417:750-754.

8. VanderLaan, P.A., Reardon, C.A., and Getz, G.S 2004. Site specificity of atherosclerosis: site-selective responses to atherosclerotic modulators [review]. Arterioscler. Thromb. Vasc. Biol. 24:12-22.

9. Van Eck, M., Bos, I.S., Hildebrand, R.B., Van Rij, B.T., and van Berkel, T.J. 2004. Dual role for scavenger receptor class B, type I on bone marrow-derived cells in atherosclerotic lesion development. Am. J. Pathol. 165:785-794.

10. Herz, J., and Hui, D.Y. 2004. Lipoprotein receptors in the vascular wall. Curr. Opin. Lipidol. 15:175-181

11. Greaves, D.R., and Gordon, S. 2005. Thematic review series: the immune system and atherogenesis. Recent insights into the biology of macrophage scavenger receptors. J. Lipid Res. 46:11-20.

12. Yoshida, H., Quehenberger, O., Kondratenko, N., Green, S., and Steinberg, D. 1998. Minimally oxidized low-density lipoprotein increases expression of scavenger receptor A, CD36, and macrosialin in resident mouse peritoneal macrophages. Arterioscler. Thromb. Vasc. Biol. 18:794-802.

13. Miller, Y.I., et al. 2003. Minimally modified LDL binds to CD14, induces macrophage spreading via TLR4/MD-2, and inhibits phagocytosis of apoptotic cells. J. Biol. Chem. 278:1561-1568.

14. Liang, C.P., et al. 2004. Increased CD36 protein as a response to defective insulin signaling in macrophages. J. Clin. Invest. 113:764-773. doi:10.1172/ JCI200419528.

15. Li, A.C., and Glass, C.K. 2002. The macrophage foam cell as a target for therapeutic intervention. Nat. Med. 8:1235-1242.

16. Kunjathoor, V.V., et al. 2002. Scavenger receptors class A-I/II and CD36 are the principal receptors responsible for the uptake of modified low density lipoprotein leading to lipid loading in macrophages. J. Biol. Chem. 277:49982-49988.
17. Khoo, J.C., Miller, E., Pio, F., Steinberg, D., and Witztum, J.L. 1992. Monoclonal antibodies against LDL further enhance macrophage uptake of LDL aggregates. Arterioscler. Thromb. Vasc. Biol. 12:1258-1266

18. Schissel, S.L., et al. 1998. Secretory sphingomyelinase, a product of the acid sphingomyelinase gene, can hydrolyze atherogenic lympoproteins at neutral $\mathrm{pH}$. Implications for atherosclerotic lesion development. J. Biol. Chem. 273:2738-2746.

19. Griffith, R.L., Virella, G.T., Stevenson, H.C., and Lopes-Virella, M.F. 1988. Low density lipoprotein metabolism by human macrophages activated with low density lipoprotein immune complexes. A possible mechanism of foam cell formation. J. Exp. Med. 168:1041-1059.

20. Yeh, M., et al. 2004. Role for sterol regulatory element-binding protein in activation of endothelial cells by phospholipid oxidation products. Circ. Res. 95:780-788.

21. Kruth, H.S., et al. 2005. Macropinocytosis is the endocytic pathway that mediates macrophage foam cell formation with native low density lipoprotein. J. Biol. Chem. 280:2352-2360.

22. Lindqvist, P., Ostlund-Lindqvist, A.M., Witztum, J.L., Steinberg, D., and Little, J.A. 1983. The role of lipoprotein lipase in the metabolism of triglyceride-rich lipoproteins by macrophages. J. Biol. Chem. 258:9086-9092.

23. Tall, A.R. 2003. Role of ABCA1 in cellular cholesterol efflux and reverse cholesterol transport. Arterioscler. Thromb. Vasc. Biol. 23:710-711.

24. Hoebe, K., et al. 2005. CD36 is a sensor of diacylglycerides. Nature. 433:523-527.

25. Kunjathoor, V.V., Tseng, A.A., Medeiros, L.A., Khan, T., and Moore, K.J. 2004. beta-Amyloid promotes accumulation of lipid peroxides by inhibiting CD36mediated clearance of oxidized lipoproteins. J. Neuroinflammation. 1:23. doi:10.1186/1742-2094-1-23.

\title{
Weaving $\beta$ Klotho into bile acid metabolism
}

\author{
Antonio Moschetta ${ }^{1}$ and Steven A. Kliewer,1,2
}

1Department of Pharmacology and 2Department of Molecular Biology, University of Texas Southwestern Medical Center, Dallas, Texas, USA.

Bile acids are natural detergents that assist in the absorption and digestion of fats in the intestine. In liver, the synthesis of bile acids from cholesterol is regulated by multiple signaling cascades that repress transcription of the gene encoding cholesterol $7 \alpha$-hydroxylase (CYP7A1), the rate-limiting enzyme in the classic bile acid synthesis pathway. In this issue of the JCI, Ito and coworkers demonstrate that mice lacking $\beta$ Klotho, a membrane protein with 2 putative glycosidase domains, have increased Cyp7a1 mRNA levels and bile acid concentrations (see the related article beginning on page 2202). $\beta \mathrm{Klotho}-\mathrm{KO}$ mice also have small gallbladders and are resistant to cholesterol gallstone formation. These findings highlight the central role of $\beta$ Klotho in bile acid homeostasis and raise the possibility that this protein could be a pharmacologic target for the treatment of gallstones.

Nonstandard abbreviations used: CYP7A1, cholesterol $7 \alpha$-hydroxylase; FGFR4, fibroblast growth factor receptor 4; FXR, farnesoid X receptor; SHP, small heterodimer partner.

Conflict of interest: The authors have declared that no conflict of interest exists.

Citation for this article: J. Clin. Invest. 115:2075-2077 (2005). doi:10.1172/JCI26046.
Bile acids are cholesterol metabolites that are synthesized in the liver, stored in the gallbladder, and released during a meal into the small intestine, where they are crucial for the absorption of lipophilic nutrients and vitamins (1). Despite their importance in normal physiology, bile acids are strong detergents whose con- centrations must be tightly controlled. Dysregulation of bile acid homeostasis is associated with a range of pathophysiological disorders including cholestatic liver disease and cholesterol gallstone formation. In this issue of the JCI, Ito et al. make an interesting and unexpected link between the protein $\beta$ Klotho and the regulation of bile acid synthesis (2).

\section{Feedback repression of bile acid synthesis}

Cholesterol $7 \alpha$-hydroxylase (CYP7A1), which is encoded by the CYP7A1 gene, catalyzes the rate-limiting step in the conversion of cholesterol to bile acids (Figure 1). Bile acids act via a feedback mechanism to repress CYP7A1 transcription. Work from a number of laboratories has revealed the complexity of this regulation $(1,3)$. One pathway through which CYP7A1 is 